\title{
Surges of advanced medical support associated with influenza outbreaks
}

\author{
J. C. KING JR. ${ }^{1 *}$, J. E. SCHWEINLE ${ }^{1}$, R. J. HATCHETT ${ }^{2}$, Y. GAO ${ }^{1}$, \\ R. LICHENSTEIN ${ }^{3}$ AND J. ZHOU ${ }^{1}$ \\ ${ }^{1}$ US Department of Health and Human Services, Assistant Secretary for Preparedness and Response, Biomedical \\ Advanced Research and Development Authority, Washington, DC 20201, USA \\ ${ }^{2}$ Coalition for Epidemic Preparedness Innovations (CEPI), CEPI, clo Norwegian Institute of Public Health, PB \\ 4404 Nydalen, N-0403 Oslo, Norway \\ ${ }^{3}$ Department of Pediatrics, University of Maryland School of Medicine, Baltimore, Maryland 21201, USA
}

Received 1 January 2017; Final revision 20 April 2017; Accepted 10 May 2017;

first published online 9 June 2017

\section{SUMMARY}

We utilized de-identified data to evaluate increases in four outcomes during influenza outbreak periods (IOPs) including: hospitalization, intensive care unit admission, mechanical ventilation or death for adults aged 18 years or older with medically attended acute respiratory illnesses

(MAARI) admitted to any of Maryland's 50 acute-care hospitals over 12 years. Weekly numbers of positive influenza tests in the Maryland area were obtained from the US Center for Disease Control and Prevention interactive website. The fewest consecutive weeks around the peak week containing at least $85 \%$ of the positive tests defined the IOP. Weekly counts of individual study outcomes were positively correlated with regional weekly counts of positive influenza tests during all the IOPs over 12 years. Also, rate ratios comparing daily occurrences of each study outcome between the IOP and non-IOP were significantly elevated. These results confirm conclusions of previous studies that influenza outbreaks are clearly associated with deaths and increased use of advanced medical resources by patients with MAARI. These data analyses suggest that increased efforts to develop more effective influenza vaccines and therapeutics should be a priority.

Key words: Death, hospitalization, influenza, intensive care unit, mechanical ventilation, medical support.

\section{INTRODUCTION}

Influenza illnesses are estimated to occur in $5-20 \%$ of the population and are a substantial cause of worldwide morbidity and mortality every year [1-4]. In addition, influenza outbreaks impose a considerable economic burden. In the USA influenza-related

\footnotetext{
* Author for correspondence: J. C. King Jr., M.D., US Department of Health and Human Services, Assistant Secretary for Preparedness and Response, Biomedical Advanced Research and Development Authority, Washington, DC 20201, USA. (Email: james.king@hhs.gov)
}

illnesses are estimated to result in costs of US\$ 87 billion annually, with over US\$ 6 billion related to direct hospital care [1,5].

Multiple publications have reported the association of seasonal and pandemic influenza outbreaks with increased utilization of advanced medical care including hospitalization, intensive care unit (ICU) admissions, mechanical ventilation or deaths as individual factors [2, 6-11]. However, simultaneous evaluation of surges of all four outcomes in a large fixed population showing their relationship to yearly influenza outbreaks over multiple years has not been reported. 
The objective of this investigation was to simultaneously evaluate temporal increases of each of these four advanced medical support outcomes during influenza outbreaks and to show their relationship to regional influenza virus surveillance data reported by the US Centers for Disease Control and Prevention (CDC) over multiple years. Information about temporal increases of these study outcomes was obtained from inpatient medical support and outcome data collected from all 50 Maryland acute-care hospitals over 12 consecutive study years. This allowed a longitudinal evaluation of the relationship between demand for medical resources and the timing of influenza seasons dominated by different influenza strains or types and of varying intensity, including the A/H1N1 pandemic (H1N1pdm) during the 2008-2009 and 20092010 seasons.

\section{SUBJECTS AND METHODS}

\section{Study data}

The study database included all adult patients admitted to any of the 50 Maryland state-regulated acute-care hospitals over 12 consecutive study years from 1 July 2001 through 29 June 2013. Importantly, these hospitals represent a spectrum of community, teaching and public acute-care medical centers [12]. Bed capacity for these acute-care hospitals ranged from 9 to 1000 and adult critical care beds from 0 to 266. The State of Maryland's Health Services Cost Review Commission (HSCRC) provided data on advanced medical support and death as well as diagnostic codes for all hospitalized patients for all 12 study years [13]. The HSCRC was established by Maryland law in 1971 and was given broad responsibility regarding public disclosure of hospital data to promote, among other factors, cost containment, access to care, and equity. Maryland Veterans Hospitals were not included in the investigation because they are not under state regulation and therefore HSCRC data were not available.

All data extracted from the HSCRC database were de-identified. Extracted data included demographic information such as patient admission date, age in years, gender and race. This database contained information on the study's four key outcomes including hospitalization, ICU admission, intubated mechanical ventilation and death during each patient's hospital stay. Current Procedural Terminology (CPT) codes $96 \cdot 70,96 \cdot 71$, and $96 \cdot 72$ were used to identify patients who received continuous mechanical ventilation.

\section{Study population}

The study population included all patients aged 18 years and older hospitalized in any of the 50 Maryland state-regulated acute-care hospitals with the possible diagnosis of influenza. Since patients with influenza infections most often present with signs and symptoms of respiratory-related illnesses, data for the population with medically attended acute respiratory illness (MAARI) or influenza as at least part of their hospital diagnoses were analyzed. Identification of patients with MAARI-related diagnoses was accomplished by using International Classification of Diseases version nine (ICD-9) codes of interest in a manner previously described [10, 14]. MAARI-related diagnoses included one or more primary or subsequent ICD-9 discharge codes for upper, middle and lower respiratory illnesses, as well as specific codes for fever, respiratory viral illness and influenza. Data on influenza testing on individual patients were not available in the database used.

\section{Study intervals of interest}

US Morbidity and Mortality Weekly Report (MMWR) designated weeks were utilized to define influenza seasons for 12 consecutive years from 20012002 through 2012-2013 [15, 16]. A study year started at the beginning day of MMWR week 27 (late June or early July) of 1 year and ended on the last day of MMWR week 26 (late June or early July) of the following year in order to capture at least one full influenza season. Influenza virus surveillance data for the 12 study years were obtained from the CDC interactive website for Health and Human Services (HHS) Region 3 that included Maryland, as well as Delaware, District of Columbia, Pennsylvania, Virginia, and West Virginia [17].

Weekly influenza positive test counts were used to define two discrete time periods for the analyses for each study year. The influenza outbreak period (IOP) consisted of the fewest number of consecutive MMWR weeks, including the peak week with at least $85 \%$ of the positive influenza tests for US Department of HHS Region 3 for each of the 12 individual seasons. The non-influenza outbreak period (NIOP) included the remaining weeks on either 'side' of the IOP for each study-defined year.

For this investigation, study year 2008-2009 began on 1 July 2008 and ended on 4 July 2009. This study year was anomalous in that it captured two distinct 
IOPs. The first IOP was the seasonal influenza outbreak from 15 February 2009 to 14 March 2009. The second IOP was the initial 'wave' of the A/H1N1pdm virus, which occurred from 7 June 2009 to 4 July 2009. Study year 2009-2010 started 5 July 2009 and ended 4 July 2010 and included the major A/H1N1pdm virus second wave with an IOP from 11 October 2009 to 7 November 2009.

\section{Data analyses}

The first descriptive analysis involved visual examination of whether surges of each of the four individual study outcomes in patients hospitalized with MAARI-related diagnoses were temporally related to increases in positive influenza tests in the Maryland vicinity (HHS Region 3). This examination was based upon weekly counts of each of the four key study outcomes in relation to simultaneous weekly counts of positive influenza tests during the IOPs for the 12 study years combined.

In addition, the strength of the relationship between MAARI-related outcomes and the intensity of the influenza epidemic was assessed. Spearman's rank correlation coefficients (CCs) were calculated between the numbers of weekly positive influenza tests and concomitant weekly counts of each of the four individual study outcomes during the IOPs over the 12 study years. CCs were also calculated for influenza A subtypes or for B strains. B strains were not differentiated between B Yamagata or B Victoria lineage. These analyses included all adults 18 years of age and older analyzed together as well as divided into three age subgroups: 18 to $<50$ years; 50 to $<65$ years; and 65 years and older.

In addition, rate ratios (RR) based on the number of daily counts of each MAARI-related outcomes were estimated between all the IOP and NIOP intervals for the 12 study years combined. A Poisson regression model was used to estimate the RR and associated $P$ values of each of the four study outcomes for the IOPs compared with NIOPs for the 12 study years combined. In addition to the IOP indicator, the regression model included admission year and month to account for seasonal variability that likely involved a mixture of factors. These factors may have included influenza, other respiratory pathogens, as well as seasonal or environmental issues that could have influenced the outcome measures of interest. The RR was considered a quantitative indicator of surge of these study outcomes during the IOP $v s$. the
NIOP. The estimation of RR was conducted for all adults and also for each of the three adult age subgroups as previously described.

All the data analyses were performed using the SAS software (version 9.3, SAS Institute).

\section{Human subject protection}

All data regarding influenza surveillance and hospitalization were examined and analyzed without personal identifiers. This study was reviewed and approved by the Institutional Review Board of the University of Maryland at Baltimore.

\section{RESULTS}

Table 1 demonstrates the demographics of 7474837 adult patients admitted to any of Maryland's acutecare hospitals over the 12 study years. More females than males were hospitalized overall. In the 18 to $<50$ years of age group, twice as many females as males were hospitalized. This female predominance was likely due to women admitted for labor and delivery. White race accounted for $61.9 \%$ of the subjects.

Using data from both Tables 1 and 2, 967767 $(12.9 \%)$ of total hospitalizations were MAARI related. Out of 967767 MAARI-related hospitalizations, $124240(12 \cdot 85 \%)$ patients were admitted to an ICU; $97446(10 \cdot 1 \%)$ received mechanical ventilation, and $56327(5.8 \%)$ died in the hospital. The percentage of total hospital admissions with MAARI-related illnesses increased with advancing age with $8.2 \%$, $13 \cdot 6 \%$, and $17 \cdot 4 \%$ of adults aged 18 to $<50,50$ to $<65$, and $>65$ years, respectively.

Figure 1 displays four subplots, one for each of the four study outcomes in relation to weekly counts of HHS Region 3 positive influenza tests for 12 consecutive influenza study years. These subplots illustrate that seasonal peaks of positive influenza tests were closely associated with simultaneous peaks of the four individual study outcomes of interest.

Table 3 shows Spearman's CCs between weekly counts of each of the four MAARI-related study outcomes and the weekly counts of all positive influenza tests in HHS Region 3 within the IOPs over the 12 study years combined for all adults and for the three age subgroups. In addition, CCs are presented for the three individual circulating influenza outbreak virus types or subtypes for the 12 study years. Positive correlations were identified for all four study outcomes for all ages combined. In addition, all CCs 
Table 1. Demographics of hospitalized patients

\begin{tabular}{lllll}
\hline \hline & All adults & 18 to $<50$ years & 50 to $<65$ years & 65 years and above \\
\hline $\begin{array}{l}\text { Total hospitalizations, } n \\
\text { Gender, } n \text { (\%) }\end{array}$ & 7474837 & 2901730 & 1726188 & 2846919 \\
$\quad$ Male & $3018159(40 \cdot 4)$ & $962476(33 \cdot 2)$ & $860905(49 \cdot 9)$ & $1194778(42 \cdot 0)$ \\
$\quad$ Female & $4456528(59 \cdot 6)$ & $1939151(66 \cdot 8)$ & $865256(50 \cdot 1)$ & $1652121(58 \cdot 0)$ \\
$\quad$ Unknown & $150(0 \cdot 0)$ & $103(0 \cdot 0)$ & $27(0 \cdot 0)$ & $20(0 \cdot 0)$ \\
Race, $n(\%)$ & & & & \\
$\quad$ White & $4625957(61 \cdot 9)$ & $1496038(51 \cdot 6)$ & $1045257(60 \cdot 6)$ & $2084662(73 \cdot 2)$ \\
$\quad$ African-American & $2386330(31 \cdot 9)$ & $1125076(38 \cdot 8)$ & $605995(35 \cdot 1)$ & $655259(23 \cdot 0)$ \\
Asian or Pacific Islander & $122157(1 \cdot 6)$ & $68285(2 \cdot 4)$ & $19090(1 \cdot 1)$ & $34782(1 \cdot 2)$ \\
$\quad$ American-Indian & $17040(0 \cdot 2)$ & $8851(0 \cdot 3)$ & $3779(0 \cdot 2)$ & $4410(0 \cdot 2)$ \\
$\quad$ Other & $305902(4 \cdot 1)$ & $195289(6 \cdot 7)$ & $48206(2 \cdot 8)$ & $62407(2 \cdot 2)$ \\
\hline \hline
\end{tabular}

Table 2. Rate ratios $(R R)^{a}$ of admissions with MAARI-related study outcomes for all 12 study years

\begin{tabular}{|c|c|c|c|c|}
\hline & All adults & 18 to $<50$ years & 50 to $<65$ years & 65 years and above \\
\hline \multicolumn{5}{|l|}{ Hospitalization } \\
\hline Number & 967767 & 237176 & 234168 & 496423 \\
\hline RR $(95 \%$ CI) & $1 \cdot 119(1 \cdot 103-1 \cdot 135)$ & $1 \cdot 145(1 \cdot 125-1 \cdot 165)$ & $1 \cdot 121(1 \cdot 100-1 \cdot 142)$ & 1. $106(1 \cdot 089-1 \cdot 123)$ \\
\hline$P$ value & $<0 \cdot 0001$ & $<0.0001$ & $<0 \cdot 0001$ & $<0.0001$ \\
\hline \multicolumn{5}{|l|}{ ICU admission } \\
\hline Number & 124240 & 24328 & 34642 & 65270 \\
\hline RR $(95 \%$ CI) & $1 \cdot 085(1 \cdot 063-1 \cdot 108)$ & $1 \cdot 121(1 \cdot 080-1 \cdot 165)$ & $1 \cdot 089(1 \cdot 052-1 \cdot 126)$ & $1 \cdot 070(1.043-1 \cdot 098)$ \\
\hline$P$ value & $<0 \cdot 0001$ & $<0 \cdot 0001$ & $<0 \cdot 0001$ & $<0 \cdot 0001$ \\
\hline \multicolumn{5}{|c|}{ Mechanical ventilation } \\
\hline Number & 97446 & 20397 & 27278 & 49771 \\
\hline RR $(95 \%$ CI) & $1 \cdot 109(1 \cdot 087-1 \cdot 163)$ & $1 \cdot 117(1 \cdot 073,1 \cdot 163)$ & $1 \cdot 123(1 \cdot 085-1 \cdot 162)$ & $1 \cdot 097(1 \cdot 068-1 \cdot 127)$ \\
\hline$P$ value & $<0 \cdot 0001$ & $<0 \cdot 0001$ & $<0 \cdot 0001$ & $<0.0001$ \\
\hline \multicolumn{5}{|l|}{ Death } \\
\hline Number & 56327 & 4989 & 10705 & 40633 \\
\hline RR $(95 \%$ CI) & $1 \cdot 125(1.097-1 \cdot 153)$ & $1 \cdot 163(1.075-1 \cdot 258)$ & $1 \cdot 131(1 \cdot 071-1 \cdot 195)$ & $1 \cdot 118(1 \cdot 086-1 \cdot 151)$ \\
\hline$P$ value & $<0 \cdot 0001$ & $0 \cdot 0002$ & $<0.0001$ & $<0.0001$ \\
\hline
\end{tabular}

${ }^{a}$ RR, 95\% CI, and $P$ value were estimated by comparing daily counts of each of the four study outcomes associated with medically attended acute respiratory illness (MAARI) between the influenza outbreak period to non-influenza outbreak periods.

for the age subgroups were positive with values that ranged from 0.282 to 0.594 with the exception of death for patients 18 to $<50$ years of age where the CC was only mildly elevated $0 \cdot 066$. CC's between death and weekly influenza-positive tests generally trended to be more positive with increasing age.

Table 3 further demonstrates that influenza virus subtypes and types were associated with a mixture of study outcome patterns. For A/H1N1 viruses, generally higher positive CCs between positive influenza tests and counts of the all outcomes except death were observed for the two younger age groups compared with the oldest group, aged 65 years and older. Correlation coefficients were generally more positive for $\mathrm{A} / \mathrm{H} 3 \mathrm{~N} 2$ and
$\mathrm{B}$ viruses than for $\mathrm{A} / \mathrm{H} 1 \mathrm{~N} 1$ virus for patients 65 years of age or older. Also, influenza B viruses were associated with relatively similar increases in CCs for the study outcomes as the influenza $\mathrm{A} / \mathrm{H} 3 \mathrm{~N} 2$ virus in these hospitalized patients.

Table 2 displays the estimated RRs for MAARI-related study outcomes during the IOPs compared with NIOPs for all 12 study years combined. For adults of all ages combined, the RRs were significantly elevated for all four study outcomes during the IOPs compared with NIOPs, with RRs ranging from 1.085 to 1.125 and all $P$ values $<0 \cdot 0001$. Also, each age subgroup had significantly elevated RRs for all four outcomes including the 

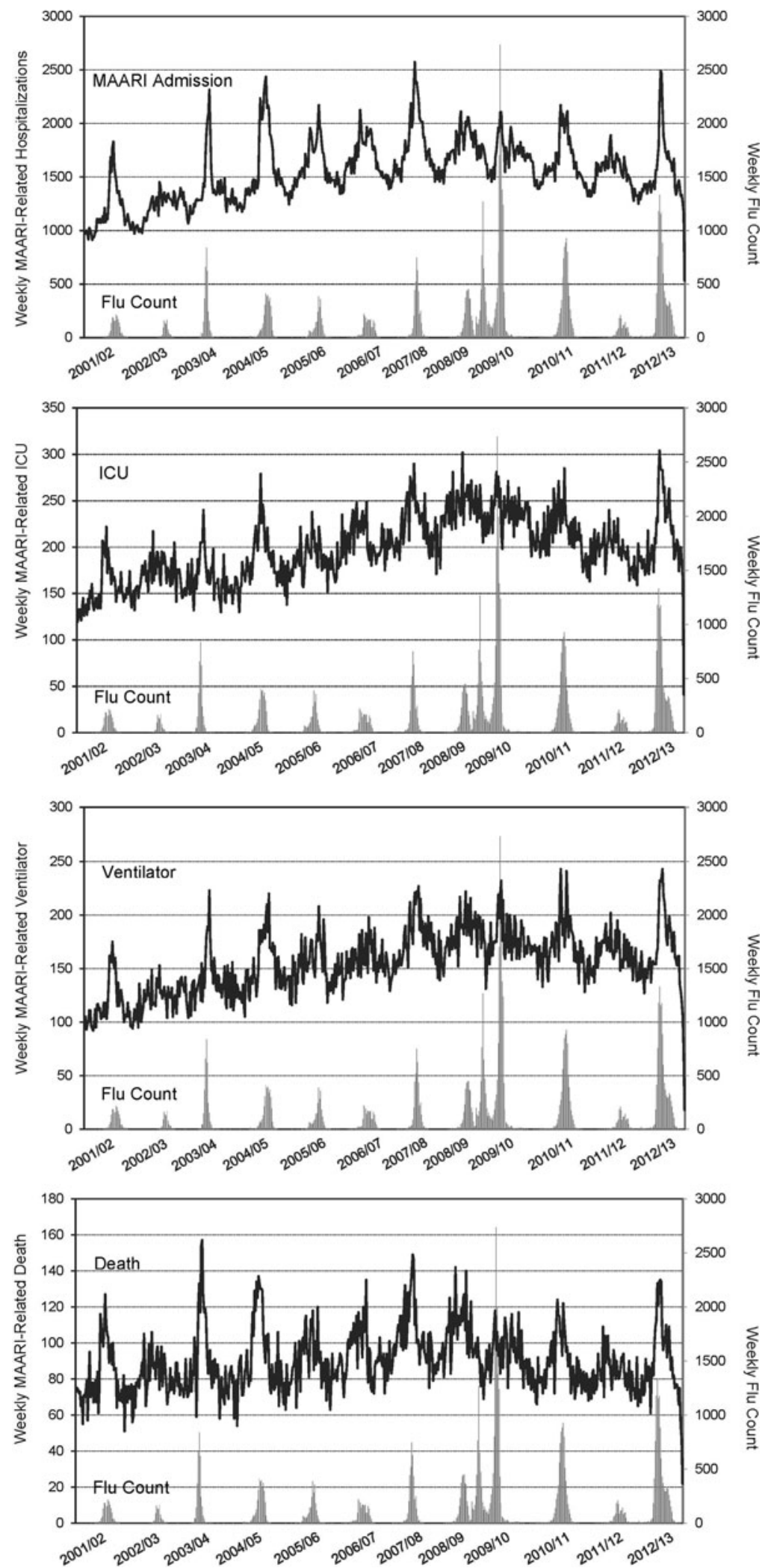

Fig. 1. Plots of the four study outcomes ${ }^{\mathrm{a}}$ and weekly counts of positive influenza tests ${ }^{\mathrm{b}}$ in the Maryland area.

${ }^{a}$ Study outcomes consist of medically attended acute respiratory illness (MAARI) related hospitalizations, intensive care unit admission, mechanical ventilation, and death.

${ }^{\mathrm{b}}$ Positive influenza test data obtained from the United States Center for Disease Control and Prevention interactive website for Region 3 [17]. 
Table 3. Spearman's correlation coefficients $(C C S)^{a}$ of the four MAARI-related ${ }^{b}$ weekly study outcomes in adults compared with weekly influenza counts within the influenza outbreak period

\begin{tabular}{|c|c|c|c|c|}
\hline Age groups & $\begin{array}{l}\text { Total flu } \\
\text { count }\end{array}$ & $\begin{array}{l}\mathrm{H} 1 \\
\text { count }\end{array}$ & $\begin{array}{l}\mathrm{H} 3 \\
\text { count }\end{array}$ & $\begin{array}{l}\text { B } \\
\text { count }\end{array}$ \\
\hline \multicolumn{5}{|l|}{ All adults } \\
\hline Hospitalization & 0.545 & $0 \cdot 245$ & $0 \cdot 359$ & $\mathbf{0} \cdot 327$ \\
\hline ICU admission & 0.598 & $0 \cdot 501$ & $0 \cdot 188$ & $0 \cdot 335$ \\
\hline $\begin{array}{l}\text { Mechanical } \\
\text { ventilation }\end{array}$ & 0.584 & $0 \cdot 384$ & $0 \cdot 381$ & $0 \cdot 349$ \\
\hline Death & $0 \cdot 382$ & $-0 \cdot 040$ & $0 \cdot 274$ & $0 \cdot 238$ \\
\hline \multicolumn{5}{|l|}{18 to $<50$ years } \\
\hline Hospitalization & $0 \cdot 456$ & $0 \cdot 213$ & 0.093 & $0 \cdot 099$ \\
\hline ICU admission & 0.563 & $0 \cdot 388$ & $0 \cdot 087$ & $0 \cdot 149$ \\
\hline $\begin{array}{l}\text { Mechanical } \\
\text { ventilation }\end{array}$ & $0 \cdot 487$ & $\mathbf{0} \cdot 317$ & $0 \cdot 201$ & $0 \cdot 099$ \\
\hline Death & 0.066 & $-0 \cdot 081$ & $-0 \cdot 112$ & $-0 \cdot 171$ \\
\hline \multicolumn{5}{|l|}{50 to $<65$ years } \\
\hline Hospitalization & 0.594 & 0.453 & $0 \cdot 332$ & $0 \cdot 318$ \\
\hline ICU admission & 0.457 & 0.522 & $0 \cdot 174$ & $0 \cdot 325$ \\
\hline $\begin{array}{l}\text { Mechanical } \\
\text { ventilation }\end{array}$ & $0 \cdot 494$ & 0.510 & $0 \cdot 327$ & $0 \cdot 306$ \\
\hline Death & $0 \cdot 282$ & $0 \cdot 303$ & $0 \cdot 213$ & $0 \cdot 146$ \\
\hline \multicolumn{5}{|l|}{$\geqslant 65$ years } \\
\hline Hospitalization & $0 \cdot 447$ & $0 \cdot 090$ & 0.509 & $0 \cdot 404$ \\
\hline ICU admission & 0.476 & $0 \cdot 316$ & $0 \cdot 227$ & $0 \cdot 320$ \\
\hline $\begin{array}{l}\text { Mechanical } \\
\text { ventilation }\end{array}$ & $0 \cdot 453$ & $0 \cdot 119$ & $0 \cdot 420$ & $0 \cdot 338$ \\
\hline Death & $0 \cdot 328$ & $-0 \cdot 156$ & 0.307 & $0 \cdot 304$ \\
\hline
\end{tabular}

${ }^{a}$ Bold CC denote moderate-to-high elevation.

${ }^{\mathrm{b}}$ MAARI is medically attended acute respiratory illness.

group of patients 18 to $<50$ years of age. Moreover, this younger age group had a trend towards similar but numerically higher RRs for three of the four outcomes (hospitalization, ICU, death) compared with the two older groups. In addition, numerically higher RRs were observed for the category death vs. the other three outcomes for all age groups combined and each of the three age subgroups.

\section{DISCUSSION}

The results of this study demonstrated that significant surges in all four study outcomes occur simultaneously with influenza outbreaks almost every influenza season in adults. These results confirm multiple previous reports of increases of the individual outcome components investigated in this study associated with influenza outbreaks [1, 2, 6-18]. The present study is unique in that it provides quantitative assessments of the association of all four outcomes to influenza outbreaks for 12 consecutive years for nearly one million patients, and estimated RR of the outcomes of interest during the IOP compared against the NIOP.

Analyses of the present study's data reaffirm previous publications on the impact of age or virus type on advanced medical outcomes related to influenza outbreaks $[19,20] . \mathrm{A} / \mathrm{H} 1 \mathrm{~N} 1$ viruses are associated with greater use of advanced medical care in the two younger age groups of 18 to 64 years compared with the group 65 years and older, but $\mathrm{A} / \mathrm{H} 3 \mathrm{~N} 2$ and $\mathrm{B}$ viruses were associated with higher $\mathrm{CC}$ s for the oldest group aged 65 years of age or older.

Second, illnesses due to influenza B have been considered to be milder than illnesses caused by influenza A viruses for uncomplicated influenza infections [18, 21]. However, the present study corroborates more recent findings that hospitalized patients with influenza B have similar severities in patients hospitalized with influenza A viruses with regard to ICU admission, mechanical ventilation or inpatient deaths [22-24].

Third, younger adults are less frequently hospitalized with influenza infection $[7,18]$. However, data reported in the present study support a previous study [2] showing that once hospitalized, younger adults aged 18 to $<65$ years, and even adults younger than 50 years of age experience similar severity of illness in terms of the percentage of hospital admissions resulting in an ICU admission, mechanical ventilation or death when compared with adults 65 years of age or older. A possible explanation for this observation is that clinicians may more frequently hospitalize older individuals with a less severe respiratory illness than young adults because of increased risk factors [25].

There are limitations in this study. First, it was not possible to directly identify the pathogens in the study population that were associated with increases in the four study outcomes. However, linking the surges in MAARI-related study outcomes to influenza surveillance suggests that influenza illness was an important factor for these outcome increases. Second, HHS Region 3 influenza virus surveillance data may not exactly match the quantity and type of influenza viruses circulating specifically in Maryland. Third, data from Maryland hospitals may not reflect influenza outcomes in other states or regions because of possible geographic and demographic differences. Finally, there are confounders that could have an impact on the estimation of the CCs and RRs, but could not be adequately evaluated by using large administrative or observational databases. However, 
linking surges of MAARI-related study outcomes to influenza surveillance suggest that influenza illness was at least an important factor for these medical resource and outcome increases.

The advantage of using the State of Maryland HSCRC data for the analyses was that it included a broad variety of hospitals [12]. Maryland acute-care hospitals included community, teaching, and public hospitals that differ in their floor and ICU bed capacity and may differ in their patient populations as well as clinician practices. This hospital variety may more realistically mirror the broad spectrum of medical care delivered to patients with more severe influenza illness.

\section{SUMMARY}

The results of this study reaffirm that surges in advanced medical support and in-hospital deaths during influenza outbreaks each season were common over a 12-year period of time. These observations strongly support recommendations that the development of more effective vaccines should be a high priority [26] and that new, more effective therapeutic agents for influenza must be found to decrease the mortality, morbidity and cost of influenza epidemics.

\section{ACKNOWLEDGEMENTS}

The authors wish to thank Robin Robinson PhD, Robert Huebner PhD and Robert Walker M.D. from BARDA for their support and editorial review. Also, they thank Oscar Ibarra; Chief of the Information Management and Program Administration for the Maryland Health Services Cost Review Commission (HSCRC) for his assistance in obtaining hospital-based data utilized in this study. No funding was utilized for this study.

\section{DECLARATION OF INTEREST}

All the authors declare that there are no conflicts of interest.

\section{ETHICAL APPROVAL}

The University of Maryland School of Medicine Institutional Review Board has reviewed and approved this study. The authors assert that all procedures contributing to this work comply with the ethical standards of the relevant national and institutional committees on human experimentation and with the Helsinki Declaration of 1975, as revised in 2008.

\section{REFERENCES}

1. Molinari NA, et al. The annual impact of seasonal influenza in the US: measuring disease burden and costs. Vaccine 2007; 25: 5086-5096.

2. Reed $\mathbf{C}$, et al. Estimating influenza disease burden from population-based surveillance data in the United States. PLoS ONE 2015; 10(3) (http://dx.doi.org/10.1371/journal. pone.0118369).

3. Cox NJ, Subbarao K. Global epidemiology of influenza: past and present. Annual Review of Medicine 2000; 51: 407-421.

4. World Health Organization. Influenza (http://www.who. int/mediacentre/factsheets/2003/fs211/en/). Accessed 12 December 2016.

5. Karve S, et al. Annual all-cause healthcare costs among influenza patients with and without influenza-related complications: analysis of a United States managed care database. Applied Health Economics and Health Policy 2013; 11: 119-128.

6. Selwyns DC. Excess mortality from causes other than influenza and pneumonia during influenza epidemics. Public Health Reports 1932; 47: 2159-2189.

7. Thompson WW, et al. Influenza-associated hospitalizations in the United States. Journal of the American Medical Association 2004; 292: 1333-1340.

8. Goldstein E, et al. Improving the estimation of influenza-related mortality over a seasonal baseline. Epidemiology 2012; 23: 829-838.

9. Schaffer A, et al. The impact of influenza A (H1N1) pdm09 compared with seasonal influenza on intensive care admissions in New South Wales, Australia, 2007 to 2010: a time series analysis. BMC Public Health 2012; 12: 869. doi: 10.1186/1471-2458-12-869.

10. King JC, et al. Surge in ventilator use during influenza outbreaks. Disaster Medicine Public Health Preparedness 2014; 8: 136-142.

11. Thompson WW, et al. Mortality associated with influenza and respiratory syncytial virus in the United States. Journal of the American Medical Association 2003; 289: 179-186.

12. Maryland Health Care Commission. Annual Report on Selected Maryland Acute Care and Special Services. Fiscal Year 2013. Effective 1 July 2012 (http://mhcc.maryland.gov/mhcc/pages/hcfs/hcfs_hospital/documents/acute_ care/con_acute_special_hospital_rpt_2013.pdf). Accessed 3 January 2017.

13. The Maryland Health Services Cost Commission (http:// www.hscrc.state.md.us). Accessed 3 January 2017.

14. Lichenstein $\mathbf{R}$, et al. The relationship between influenza outbreaks and acute ischemic heart disease in Maryland residents over a 7-year period. Journal of Infectious Diseases 2012; 206: 821-827.

15. MMWR Weeks-CDC (https://wwwn.cdc.gov/nndss/ document/MMWR_week_overview.pdf). Accessed 3 January 2017.

16. New Mexico's Indicator-Based Information System (NM-IBIS). MMWR Week Description and Corresponding Calendar Dates (2006-2020) (https://ibis. health.state.nm.us/resource/MMWRWeekCalendar.html). Accessed 3 January 2017. 
17. Center for Disease Control and Prevention. Interactive site: National and Regional Level Outpatient Visits and Viral Surveillance (http://www.cdc.gov/flu/weekly/ fluviewinteractive.htm). Accessed 3 January 2017.

18. Simonson L, et al. The impact of influenza epidemics on hospitalizations. Journal of Infectious Diseases 2000; 181: 831-837.

19. Chaves SS, et al. Patients hospitalized with laboratoryconfirmed influenza during the 2010-2011 influenza season. Journal of Infectious Diseases 2013; 208: 1305-1314.

20. Armstrong GL, Brammer L, Fineli L. Timely assessment of the severity of the $2009 \mathrm{H} 1 \mathrm{~N} 1$ pandemic. Clinical Infectious Diseases 2011; 52(S1): S83-S89.

21. Centers for Disease Control and Prevention. Epidemiology of vaccine preventable diseases (http:// www.cdc.gov/vaccines/pubs/pinkbook/flu.html). Accessed 1 November 2016.

22. Su S, et al. Comparing clinical characteristics between hospitalized adults with laboratory-confirmed influenza
A and B virus infections. Clinical Infectious Diseases 2014; 59: 252-255.

23. Center for Disease Control and Prevention. Don't take influenza B lightly in adults (http://www.cidrap.umn. edu/news-perspective/2014/os/cdc-dont-take-influenza-blightly-adults). Accessed 3 January 2017.

24. Gutiérrez-Pizarraya A, et al. Unexpected severity of cases of influenza B infection in patients that required hospitalization during the first post pandemic wave. Journal of Infection 2012; 65: 423-430.

25. Mandell LA, et al. Infectious Disease Society of America/Thoracic Society Consensus guidelines on the management of community-acquired pneumonia in adults. Clinical Infectious Diseases 2007; 44: S27-72.

26. Donabedian A. The Quest for More Effective Influenza Vaccines (https://www.medicalcountermeasures.gov/media/ 36865/donabedian_quest-for-more-effective-influenzavaccines-bid2105-508.pdf). Accessed 3 January 2017. 